Corps et culture

Numéro 3 | 1998

Sport et lien social

\title{
Société ou communauté. Tribalisme et sentiment d'appartenance
}

\section{Michel Maffesoli}

\section{(2) OpenEdition}

1 Journals

Édition électronique

URL : http://journals.openedition.org/corpsetculture/520

DOI : 10.4000/corpsetculture.520

ISSN : $1777-5337$

Éditeur

Association Corps et Culture

Édition imprimée

Date de publication : 1 juin 1998

ISSN : 1268-5631

Référence électronique

Michel Maffesoli, «Société ou communauté. Tribalisme et sentiment d'appartenance », Corps et culture [En ligne], Numéro 3 | 1998, mis en ligne le 12 octobre 2007, consulté le 08 septembre 2020 URL : http://journals.openedition.org/corpsetculture/520 ; DOI : https://doi.org/10.4000/ corpsetculture.520

Ce document a été généré automatiquement le 8 septembre 2020

(c) tous droits réservés 


\title{
Société ou communauté. Tribalisme et sentiment d'appartenance
}

\author{
Michel Maffesoli
}

Introduction

1 Il va s'agir ici d'une mise en perspective socio-anthropologique, plus que sociologique stricto sensu. En effet, les importants changements de valeurs qui son en train de s'opérer dans nos sociétés en cette fin de siècle obligent à prendre du recul. C'est ce que l'on peut faire en restant dans un premier temps assez général. On peut ensuite proposer un levier méthodologique pour tenter de comprendre ces changements de valeurs auxquels l'on vient de faire allusion. En utilisant pour cela une métaphore, celle de tribalisme, phénomène qui serait de nos jours résurgent, entraînant toute une série de conséquences pour la constitution même de l'individu.

Éléments anthropo-sociologiques de base

2 Pour dresser très rapidement le cadre dans lequel je vais proposer mes hypothèses, il faut rappeler quelques données, au demeurant banales, de la modernité.

3 Il y a tout d'abord la notion d'épistémè. A travers cette notion Michel Foucault indique que finalement nous sommes plus pensés que nous pensons, et plus agis que nous agissons. En proposant au débat la notion d'épistémè, Michel Foucault veut dire que les formes de représentation et d'organisation sociale ont un double aspect: d'un côté, quelque chose qui va façonner en profondeur et de manière souterraine les représentations sociales, d'un autre côté le fait que ces représentations sociales ont elles-mêmes toute une série de conséquences sur l'organisation sociale, quoique cela ne soit pas forcément pensé, conscientisé ou verbalisé en tant que tel. On peut en dire autant des découvertes scientifiques. C'est ainsi qu'un auteur comme Thomas Kuhn faisait les mêmes constatations que Michel Foucault. A travers la notion de paradigme (plus souple que celle de modèle), il montre bien comment existe une matrice à partir de laquelle croissent, dans le domaine scientifique, les manières de se représenter le monde.

4 La notion de bassin sémantique proposée par Gilbert Durand s'inspirant de l'inconscient collectif de Jung, est également une notion intéressante à considérer. 
Gilbert Durand utilise l'image du bassin sémantique pour indiquer comment de petites choses vont donner les choses plus importantes. Le ruissellement finit par donner le fleuve en bas de la vallée, fleuve que l'on va nommer, que l'on va canaliser, qui va finalement se perdre dans le delta, puis se jeter dans la mer, jusqu'à ce qu'un nouveau cycle recommence.

De la même manière, on peut évoquer une nappe phréatique qui, quoique nous ne la voyons pas, sustente en profondeur la vie, c'est-à-dire nos manières d'être.

6 C'est ce que l'on peut appeler une ambiance sociale, recouvrant la notion d'esprit du temps, dont Hegel au XIX ${ }^{e}$ siècle a montré l'importance. Ainsi, avant même la constitution des individus ou des acteurs sociaux membres de la société, il y a quelque chose qui dépasse tout un chacun, qui dépasse la globalité de la société. Ce quelque chose est à vrai dire " mystérieux », dans le sens le plus simple de ce terme, c'est-à-dire ce qui fait lien, qui unit les gens entre eux.

7 C'est ici une autre manière de désigner la culture, non pas les grandes oeuvres qui constituent la culture, mais ce substrat, ce «background » dans lequel chacun baigne sans y faire attention. C'est au fond ce que l'on suce avec le lait maternel, ce dont l'éducation nous imprègne. Que ce soit celui de l'université, il y a là une culture qui nous façonne. En même temps cette notion d'épistémè n'est pas une donnée particulière aux temps modernes. On peut l'appliquer à bien d'autres périodes, selon une évolution cyclique qui doit être prise en compte pour bien apprécier la pertinence de ce propos. Mais alors la question se pose, du passage d'une épistémè à une autre.

8 Le passage, l'achèvement d'une épistémè et le commencement d'une autre, est une question difficile, qu'on ne peut penser qu'avec beaucoup de prudence. On peut retenir une proposition faite par Sorokin, sociologue américain de la culture. Cet auteur utilise la notion de saturation. Il montre par là, comment, à un moment donné, un ensemble culturel perd de son évidence. C'est précisément lorsque cette évidence se perd qu'on va entrer progressivement dans un autre type d'épistémè. Un peu comme dans la relation amicale ou amoureuse, l'évidence de l'amour, l'évidence de l'amitié, disparaît un beau matin, sans qu'on puisse dire précisément pourquoi. Simplement, on évoquera une lassitude, une usure qui vient en somme de l'histoire commune longtemps partagée avec quelqu'un, comme si tout devait, quasi mécaniquement, se fatiguer.

On peut se demander si ce n'est pas quelque chose de cet ordre qui est actuellement en jeu dans nos sociétés, derrière ce que l'on nomme communément la crise.

10 On ne sait pas très bien ce qu'il faut mettre sous ce terme de crise, sinon qu'on s'accorde de plus en plus à reconnaître que cette crise n'est pas ou n'est plus seulement économique, qu'elle n'est pas non plus uniquement politique, qu'elle n'est pas davantage simplement culturelle. Sans doute vaut-il mieux parler ici d'une perte d'évidence. Cela signifie alors qu'à un moment donné, un ensemble social, ou mieux civilisationnel n'a plus conscience de ce qu'il est. Il ne sait plus quels sont les grands mythes qui l'animent. Il n'a plus confiance en ce qu'il est. Songeons ici, à une formule de Kundera dans L'insoutenable légèreté de l'être: «il en est des amours comme des empires, lorsque cesse l'idée sur laquelle ils reposent, ils disparaissent avec elle ».

11 A bien des égards, on peut penser que le mythe qui avait été à la base de a modernité s'est saturé. Ce qui a constitué, jusqu'à maintenant, l'ossature sur a base de laquelle se sont organisées les représentations, sur la base de laquelle s'est structurée la société, s'est en quelque sorte épuisé, au point que quelque chose d'autre peut naître. 
Le mythe de la modernité

Il est important de se référer une nouvelle fois à Sorokin. Cet auteur indique, en effet, qu'il existe empiriquement deux types d'ensembles sociaux

- des ensembles sociaux plutôt de type rationaliste,

- des ensembles sociaux plutôt de type sensualiste.

Nietzsche avait, à sa manière, formulé cette dichotomie lorsqu'il avait souligné le balancement entre Apollon et Dionysos. Des historiens de l'art ont aussi repris ces distinctions ainsi Walter Pater. De même, le sociologue Karl Mannheim faisait le même type de proposition. Pour ma part, j'ai également réutilisé ces notions pour souligner que quelque chose aurait constitué la modernité autour de la figure emblématique d'Apollon. Cependant d'un point de vue sociologique, je préfère Prométhée, celui qui vole le feu aux Dieux, celui à partir duquel vont se reconnaître un certain nombre d'attitudes actives en soi et sur le monde. L'hypothèse est que cette figure emblématique d'Apollon ou de Prométhée laisse actuellement subrepticement, ou d'une manière plus ou moins affichée, la place à la figure emblématique de Dionysos.

Autrement dit, une société plus sensualiste remplace peu à peu la société rationaliste qui fut la société moderne. En effet, la figure emblématique de Prométhée ou d'Apollon, donc tout ce qui va caractériser la modernité, s'organise autour de quelques grands mots-clés.

Quels pourraient être, maintenant, les mots-clés de la modernité ?

La conception du temps est une conception du temps finalisé. élément de la triade temporelle, cet ensemble social met l'accent: sur le passé, sur le présent ou sur l'avenir

17 On repère des sociétés pour lesquelles le temps va être tourné vers le passé c'est le cas des sociétés dites traditionnelles, pour lesquelles rien ne s'innove, tout se fait en référence à la tradition, c'est-à-dire à ce qui est déjà passé.

Au contraire, d'autres sociétés font reposer l'accent sur le présent.

Il en est d'autres encore pour lesquelles tout va se constituer en fonction de l'avenir. La société moderne a fonctionné ainsi, en référence à l'avenir, avec, comme conséquence, le mythe du progrès, expression majeure de ce temps finalisé.

Il est très difficile de savoir pourquoi nos sociétés se sont ainsi orientées dans cette conception du temps finalisé. On ne peut que le constater, à partir du XVII ${ }^{\mathrm{e}}-\mathrm{XVIII}^{\mathrm{e}}$ siècle, et plus encore au XIX ${ }^{e}$ siècle, où cette orientation de la société en fonction de son avenir va atteindre son acmé.

21 Toute la philosophie de l'histoire hégélienne, toutes les diverses analyses des philosophes ou des sociologues, tel Auguste Comte, sont ainsi faites en fonction de ce qui est à venir. Aujourd'hui ne vaut qu'en fonction de demain. C'est aussi ce qu'indique Freud dans son « report de jouissance ».

22 Ce concept du temps finalisé, du temps pensé toujours en fonction de l'avenir est un des premiers éléments de la constitution de l'épistémè de la modernité. On peut dire qu'on est ici dans une conception projective : le projet est constitutif de l'individu. De la même manière, il est constitutif de l'ensemble social en son entier.

23 Le vecteur de ce temps finalisé, le fait qu'on ne va penser le monde qu'en fonction du futur, c'est une manière d'accentuer la raison. 
La rationalité

Bien sûr, la rationalité est une des caractéristiques de l'animal humain, mais à certains moments de son histoire, cette rationalité prend chez l'homme, une orientation spécifique. Je me réfère ici aux philosophes de l'Ecole de Francfort qui ont bien montré, comment, au XIX ${ }^{e}$ siècle, la rationalité devenait rationalisme, En allemand, le terme est plus significatif. La Sweckrationalität est une raison qui s'oriente en fonction d'un but. Selon cette perspective, "n'a de sens, que ce qui a du sens »; du même coup devient insensé, ce qui n'a pas du sens. De ce point de vue la polysémie du terme en français est intéressante car elle permet de souligner qu'on ne va retenir ici, au sein de cette grande capacité humaine qu'est la raison, qu'un type même de cette raison, quelque chose qui ne donne sens qu'en fonction du futur. Cela a été traduit par l'expression « rationalité instrumentale ", ce qui exprime bien cette conception de la modernité qui veut que toute chose ne vaut que dans la mesure où elle sert, où elle repose sur l'utilité. On peut ici se référer à Heidegger lorsqu'il parle de "l'ustensilité ». A partir de là, toute une série de conséquences sociales mériteront attention.

La maîtrise

Le troisième élément de la construction de ce mythe de la modernité c'est la maîtrise, c'est-à-dire une forme de logique de domination, sur soi et sur le monde.

La grande conception utilitaire du monde que chacun acquiert progressivement dans l'éducation, dans la socialisation, c'est le fait qu'il convient de se maîtriser. C'est cela la conception économique de la modernité : économie de soi, économie du monde. Il s'agit pour l'individu d'apprendre à avoir une identité maitresse d'elle-même et, par voie de conséquence, apprendre avec d'autres, qui sont arrivés à cette même maîtrise, à dominer le monde. Il est intéressant de rappeler cette formule de Corneille dans Cinna, précisément à une époque qui est le début même de la modernité. "Cinna », en effet, dit: "Je suis maître de moi, comme de l'Univers, je le suis, je veux l'être ». Les poètes ont souvent ainsi la capacité de bien cristalliser le temps.

Le pivot de ce schéma de la modernité, qui est de l'ordre de l'évidence, c'est l'individu ou l'individualisme.

L'individualisme

28 L'individualisme apparaît en quelque sorte comme l'expression théorique de la modernité. Il s'inscrit dans son cadre général. La principium individuationis est certainement le point essentiel à partir duquel se pense la modernité, à partir duquel nous élaborons tous nos systèmes. Mais le resituer ainsi au sein de la modernité, nous indique que c'est un phénomène ponctuel, il n'a pas toujours existé; il n'existera pas forcément toujours. On peut dire que ce principium individuationis est saturé dans les faits, alors qu'il n'est pas dans nos têtes, tout au moins dans les têtes de l'intelligentsia, c'est-à-dire de ceux qui sont dans le pouvoir de faire et dire quelque chose.

On peut rapidement faire une généalogie de ce principe d'individuation.

Quand Descartes pose son cogito ergo sum, il indique bien, par rapport à ce qu'était la pensée collective du Moyen-âge, qu'il n'y a de pensée qu'individuelle. D'ailleurs la formule latine dans son entier est plus intéressante : "cogito ergo sum in arcem meum ", « je pense donc je suis, dans la forteresse de mon esprit». Cette formule montre bien «l'enclosure » spécifique qui est celle de la constitution de l'individu au début de la modernité. Elle montre bien aussi cette différence fondamentale par rapport à une 
pensée antérieure qui était collective. Et, il n'est pas impossible que nous retournions ultérieurement à une pensée collective.

31 La Réforme, un peu dans le même temps que le cartésianisme, introduit également quelque chose de l'ordre de l'individualisme, car avec elle la relation à la déité n'est plus affaire collective. Luther et Calvin introduisent la notion d'un libre arbitre, l'expression d'un rapport individuel qu'un « je » va établir avec l'altérité absolue.

Jean-Jacques Rousseau fera la même chose. C'est bien à partir d'un individu rationnel que peut se penser un contrat social, comme cela apparaîtra avec la révolution française et le code napoléonien. Finalement le maître mot suite à ce que j'ai dit de Descartes, de la Réforme et de la philosophie des Lumières, c'est bien l'autonomie, l'individu autonome. Rappelons l'étymologie du terme «autonomos»: je suis ma propre loi. Il me semble que c'est là que se situe le pivot de la modernité, dans cette conception de l'individu qui se donne sa loi à lui-même, et qui ensuite peut s'associer avec d'autres individus autonomes pour faire l'histoire. C'est à partir de là que se construit le contrat social, (dont on voit bien la fragilité de nos jours), en la citoyenneté, qui est sans doute tout ce qui fonde l'idéal démocratique. Arendt a bien montré tout ce que cet idéal doit à l'individu.

Le tribalisme

33 Par opposition à ce schéma qui vient d'être exposé, par opposition aux figures emblématiques de la modernité, ma proposition, empirique, est que l'on est désormais confronté à une hétérogénéisation de ce modèle monothéiste qui a été jusqu'à maintenant de la modernité.

1 - Il n'y a pas de théorisation de ce qui est en train de se passer dans nos sociétés occidentales, mais sociologues et anthropologues, nous sommes obliges de constater une saturation, une fatigue, une porosité de l'État-nation. Cet État-Nation, expression politique du contrat et de l'idéal démocratique se sature, et l'on pourrait rassembler toute une série d'éléments qui le démontrent, que ce soit d'une manière violente et sanglante même, dans certains pays, ou que ce soit d'une manière plus policée et plus douce dans d'autres pays.

2 - Il en est de même des grandes institutions qui se sont constituées essentiellement au XIX ${ }^{e}$ siècle: la famille, l'institution médicale, l'institution universitaire, voire l'institution ecclésiastique (l'église catholique n'a trouvé l'achèvement de sa figure qu'au concile de Vatican 1er en 1871, concile qui a concrétisé en somme la «romanisation» de l'Eglise). Ainsi, toutes les institutions sociales, quelles qu'elles soient, deviennent de plus en plus poreuses de nos jours.

On peut évoquer, pour faire image, un tissu social qui demeure entier, mais qui est en train de se miter de toute part. Ce "mitage» génère dans les institutions, les entreprises, les milieux de l'éducation, le travail social, des entités microscopiques ; que ce soient des chapelles, des microgroupes, ou mieux ce que j'appelle des tribus.

3 - Un autre élément de cette hétérogénéisation du modèle de la modernité, réside dans une saturation comparable des certitudes idéologiques d'autrefois. La pensée a été animée par de grandes idées, "ces grands récits de référence» (pour reprendre une expression de Jean-François Lyotard que pouvaient être le marxisme, le freudisme à certains égards, le Positivisme). Ces grandes idées se saturent à leur tour, deviennent de plus en plus poreuses. Ce n'est pas qu'elles n'existent plus, mais elle n'existent qu'à l'état de rémanences, et non comme références par rapport auxquelles on devrait se situer. On est aujourd'hui confronté à une sorte de patchwork, de bric-à-brac 
idéologique, à des idéologies portatives, multiples et diverses, à une forme de «babélisation de la pensée ».

4 - C'est dans le cadre de cette hétérogénéisation, ou de cette hypothèse d'hétérogénéisation politique, institutionnelle, idéologique, qu'il paraît nécessaire de mettre en question ce que j'ai défini tout à l'heure comme le pivot de la modernité : c'est-à-dire l'individu, avec son identité spécifique, sexuelle, idéologique, professionnelle.

Désormais, il semble que l'individu doive laisser la place à autre chose. Le terme reste à trouver. Pour ma part, j'ai proposé celui de "personne ", au sens étymologique du terme (persona). Cela signifie que l'on est confronté à des "masques", et que nous avons moins une identité que des identifications. L'acquisition de l'identité était jusqu'à aujourd'hui le sommet de l'éducation, l'apogée de la socialisation. Mais, nous assistons maintenant à un passage de l'identité aux identifications multiples. C'est ce passage là qui me paraît fonder la naissance, peut-être vaut-il mieux dire la renaissance, de formes tribales d'existence. Le tribalisme est ainsi une métaphore utile pour essayer, provisoirement, de prendre acte de la saturation dont il a été question, et du fait que l'individu ou l'individualisme s'estompe désormais au profit de micro-ensembles, de formes communautaires. On a trop souvent l'habitude d'insister de nos jours sur l'individu ou l'individualisme. En fait, maintenant prévalent des "affinités électives " qui ne sont plus le fait de quelques uns, mais qui sont le fait d'un assez grand nombre et qui nous constitue en tribus au sein de nos institutions. C'est frappant dans le monde universitaire. Ça l'est, tout autant dans l'ordre religieux où l'évolution sectaire est une évidence. En fait, toutes les institutions se fragmentent en entités microscopiques.

C'est là où l'idée d'autonomie qui était constitutive de la modernité laisse place à autre chose. On pourrait parler d'hétéronomie, désignant par là le fait que je ne suis plus ma propre loi. Ma loi c'est l'autre. Je n'existe que dans et par l'esprit de l'autre, que dans et par le regard de l'autre.

Le « devenir mode du monde » est à cet égard intéressant : mode vestimentaire, mode langagière, mode corporelle, mode sexuelle. il y a, comme dans le phénomène mode, quelque chose qui s'établit dans notre société, ne reposant Plus sur la volonté, mais reposant sur la contamination. C'est quelque chose de l'ordre de la viralité. La mode c'est du viral; cela donne lieu à des épidémies. Il y a à mon sens quelque chose de cet ordre qui se met en place dans tous les domaines. La pensée elle-même n'échappe pas à ce phénomène. Cela est particulièrement frappant. Là où l'on devrait penser par soimême, on voit se constituer de Petites entités, sectaires, fanatiques, s'opposant les unes aux autres.

41 A l'opposé du contrat, avec l'aspect rationnel, volontaire que comporte cette notion, est en train de se constituer une autre manière d'être, une autre forme de socialité. Cette autre manière d'être va réinvestir des éléments que l'analyse sociale avait laissés de côté : l'émotionnel et l'affectuel. L'affectuel et l'émotionnel ce n'est pas uniquement de l'ordre de l'émotif ou de l'affectif, mais bien une ambiance spécifique reposant sur les processus de contamination, sur le fait que toute une série de «transes" macroscopiques parfois, microscopiques souvent, constituent le terreau de la vie sociale.

42 En ce sens, l'homo politicus ou l'homo economicus, va de plus en plus laisser la place, pour le meilleur et pour le pire, à un homo estheticus. Ce dernier va se constituer sur les émotions partagées. C'est dans les capacités à mettre en jeu l'émotionnel, l'affectuel, 
qu'il va nous falloir désormais penser cet homo estheticus, base de ce que j'appelle le tribalisme.

\section{BIBLIOGRAPHIE}

Les thèmes de cet article sont développés essentiellement dans :

Maffesoli M. (1982) L'Ombre de Dionysos. Contribution à une sociologie de l'orgie. Paris, Librairie Méridiens/Klincksieck. Réédition (1991), Le Livre de Poche, Biblio/essais.

Maffesoli M. (1988) Le Temps des tribus. Le déclin de l'individualisme dans les sociétés de masse. Paris, Librairie Méridien/Klincksieck. Réédition (1991), Le Livre de Poche, Biblio/essais.

Maffesoli M. (1990) Au creux des apparences. Pour une éthique de l'esthétique. Paris, Pion. Réédition (1993), Le Livre de Poche, Biblio/essais.

Maffesoli M. (1997) Du nomadisme. Vagabondages initiatiques. Paris, Le Livre de Poche, Biblio/essais. Bibliographie suggérée par la rédaction

Arendt H. (1945) Le Système totalitaire. Paris, Seuil (1975).

Comte A. (1844) Discours sur l'esprit positif. Paris, U.G.E. 10/18, (1963).

Durand G. (1964) L'imagination symbolique. Paris, RU.F.

Durand G. (1969) Les Structures anthropologiques de l'imaginaire. Introduction à une archétypologie générale. Paris, Bordas.

Durand G. (1982) L'Ame tigrée. Les pluriels du Psyché. Paris, Denoël.

Durand G. (1994) L'imaginaire. Paris, Hatier.

Foucault M. (1969) L'Archéologie du savoir. Paris, Gallimard.

Freud S. (1967) La Technique psychanalytique. Paris, PUF

Freud S. (1967) Introduction à la psychanalyse. Paris, Payot.

Freud S. (1971) Cinq psychanalyses. Paris, PUF

Heidegger M. (1958) Essais et conférences. Paris, Gallimard.

Heidegger M. (1962) Chemins. Paris, Gallimard.

Heidegger M. (1971) Nietzsche. Paris, Gallimard.

Hegel (1968) Principes de la philosophie du droit. Paris, Gallimard.

Jung C.-G. (1964) L'Homme et ses symboles. Paris, Robert Laffont.

Jung C.-G (1980) Mystérium conjonctionis. Paris, Albin Michel.

Jung C.- G (1990) L'Ame et le soi. Renaissance et individuation. Paris, Albin Michel.

Kuhn T.S. (1982) La Structure des révolutions scientifiques. Paris, Flammarion.

Lyotard J.-F (1979) La Condition postmoderne. Paris, Minuit. 
Mannheim K. (1956) Idéologie et utopie. Paris, M. Rivière,

Sorokin P. (1957) Social and cultural dynamics. Boston, Porter Sargent

Sorokin P. (1959) Tendances et déboires de la sociologie américaine. Paris, Aubier.

\section{AUTEUR}

MICHEL MAFFESOLI

Professeur à la Sorbonne 